\title{
Effect of Static and Dynamic Stretching Warm-Up Methods on Agility, Speed and Leg Power Performance in School Level Netball Players
}

\author{
H.G.J.H Gnanawardena ${ }^{1}$ (D) $\triangle$, W.M.N.S Wijethunga ${ }^{2} 8$ (D) and S. Weerasinghe ${ }^{3}$ (D) \\ ${ }^{123}$ Department of Sports Science, Faculty of Applied Sciences, University of Sri Jayewardenepura, Sri Lanka \\ $\triangle$ Corresponding Author: H.G.J.H Gnanawardena, E-mail: janathri.123@gmail.com
}

\section{ARTICLE INFORMATION}

Received: April 08, 2021

Accepted: May 14, 2021

Volume: 1

Issue: 1

DOI: $10.32996 /$ jspes.2021.1.1.5

\section{KEYWORDS}

Sequence Stretch, Netball Players, Lower Body

\section{ABSTRACT}

A warm-up helps the individuals to prepare themselves for strenuous workouts. The warm-up is generally executed before participation in any physical activity or sports. The objective of the study was to investigate the effects of sequencing lower-body static and dynamic stretching combinations on agility, speed, and leg power of school-level female Netball players. Three different stretching protocols were performed: (a) Static Stretching combined with Dynamic Stretching (SS+DS), (b) Dynamic Stretching combined with Static Stretching (DS+SS), and (c) Dynamic Stretching combined with Dynamic Stretching (DS+DS). A control warm-up condition without stretching was implemented with a prior aerobic warm-up followed by dynamic activities. Dependent variables included a 30-m Sprint, Agility run, and jump tests to measure speed, agility, and leg power performance. The level of significance was set at $5 \%$ for statistical analysis. The method used for statistical analysis was twoway ANOVA. There was no significant impact of stretching protocols on agility $(p=0.257)$, speed $(p=0.106)$, and leg power $(p=0.902)$ of school-level female netball athletes. The results of the analysis allow the authors to retain the hypothesis that a sequence of static and dynamic stretching combinations does not significantly affect the agility, speed, and leg power of school-level female netball athletes.

\section{Introduction}

Warming up sessions commonly consist of activities such as slow jogging, cycling, swimming and stretching (Ab Malik, 2018). Warm-up is generally promoted as an activity that enhances athletes' performance. It is especially important in team sports demanding acceleration, deceleration, or direction changes ( Pojskic, et al., 2015). Active warm-up includes low-intensity exercises, whereas passive warm-up includes external heat resources that are useful to enhance body temperature (Ahsan \& Mohammad, 2018).

Traditionally, stretching has been a main element of the pre-event warm-up. There is a variety of stretching techniques, such as; static stretching, ballistic stretching, and passive stretching. Pre-event static stretching has been prescribed to prevent injury by increasing the range of motion about a joint or series of joints and improving dynamic activities. Dynamic stretching has recently been prescribed by strength and conditioning professionals for pre-event stretching. This increase in prescription is due to recent evidence suggesting that pre-event static stretching negatively affects performance measures, such as strength, jumping, and sprint performance. A dynamic warm-up has been shown to improve knee joint position sense, increase oxygen uptake, lower lactate concentration, raise blood $\mathrm{pH}$, improve the efficiency of thermoregulation, and improve performance for bicycle sprints and vertical jumps (Macmillian, Moore, Hatler, \& Taylor, 2006).

Sporting events involve various modes of movement. The warm-up is important to prepare the body for physical activity. There is limited scientific evidence supporting one warm-up protocol over another warm-up protocol. There is no proper guidance to confirm the suitable warm-up protocol for the training sessions. Warm-up protocols are based on the experience of individual coaches, trainers, and athletes. Multiple bio-motor abilities involve with the performance of team sports activities. Agility, speed,

\begin{tabular}{lll|l} 
K & C & AL-KINDI CENTER \\
$\mathbf{R}$ & $\mathbf{D}$ & FOR RESEARCH AN
\end{tabular} $\mathbf{R}$ D DEVELOPMENT Your gateway to world-class research

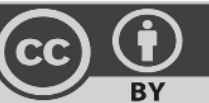

Published by Al-Kindi Center for Research and Development, London, United Kingdom. Copyright (c) the author(s). This open access article is distributed under a Creative Commons Attribution (CC-BY) 4.0 license 
and power are the major bio-motor abilities that could impact the performance of a ball game like netball. The warm-up protocol differs from another with the changes of the content of the stretching part of the warm-up protocol. Identifying the suitable stretching protocol that could enhance major bio motor-abilities performance is a challenge. The main objective of this research study is to identify the effect of sequencing lower-body static and dynamic stretching combinations (SS+DS, DS+SS, DS+DS) on agility, speed, and leg power performance of school-level female netball players.

\section{Literature Review}

Warming up before main activities is a common practice in sports. A warm-up helps the individuals to prepare themselves for strenuous workouts. Warm-up is generally executed before participation in any physical activity or sports ( Ahsan \& Mohammad, 2018). Warming up sessions commonly consist of activities such as slow jogging, cycling, swimming, and stretching. An optimal warm-up before any physical activity may help athletes in their mental and physical readiness, injury prevention, and overall sports performance (Malik, et al., 2018).

There is a variety of stretching techniques, such as; static stretching, ballistic stretching, passive stretching, and proprioceptive neuromuscular facilitation. Stretching can be done according to two ways. It can be either active or passive (Ogura, 2007). Static stretching is used to stretch muscles and slowly lengthen a muscle to a comfortable position. Numerous investigations have shown the potential for acute, static stretching to degrade performance on vertical jumps, short sprints, tasks requiring maximal voluntary contractions, muscle strength-endurance performance, balance challenges, and reaction time (McMillian, 2006). Additionally, several studies now indicate that pre-exercise static stretching does not benefit injury risk reduction (McMillian, 2006).

Professionals are increasing their support of dynamic stretching as the most effective way to prepare the athlete for the demands of their sport. Dynamic stretching comprises movements that are similar to those in which the participant will engage. McMillian et al. (2006) found that dynamic warm-ups demonstrated an improvement in power and agility measures compared to static stretch and no warm-up protocols. Dynamic stretching has recently been prescribed by strength and conditioning professionals for pre-event stretching. This increase in prescription is due to recent evidence suggesting that pre-event static stretching harms some measures of performance, such as strength, jumping and sprint performance. McMillian, et al. (2006) stated that dynamic warm-up is useful to improve knee joint position sense, increase oxygen uptake, lower lactate concentration, raise blood $\mathrm{pH}$, and improve the efficiency of thermoregulation, and to improve performance for bicycle sprints and vertical jumps. United States army physical fitness school developed a dynamic warm-up for individuals and military units and founded dynamic warm-up help to increase body temperature and heart rate, the pliability of joints and muscles, and responsiveness of nerves and muscles in preparation for physical readiness to training activities. The dynamic warm-up was used before each exercise session as part of an intervention to decrease injuries and to improve physical performance among soldiers in a basic training battalion (McMillian, et al., 2006).

Netball is a dynamic, fast, skillful, and predominantly female team sport. Many of the netball skills involve explosive movements, quick changes of directions, different types of passes, and various ways to receive and dispose of the ball within a 3-s time frame. (Hopper, et al., 1992).

Plisk (2000) defined agility as 'the ability of the body or body parts to explosively brake, change direction, and accelerate again rapidly under control'. Agility and power activities use stored energy from the stretch-shortening cycle (Plisk, 2000). In a study conducted with soccer players, Khorasani and Bafghi (2013) showed that agility contributes to some of the total distance covered during a game that determines winning possession of the ball and scoring goals in soccer (Khorasani \& Bafghi, 2013). In this study, agility was evaluated using the Illinois agility tests and the best score of three trials was recorded for each fitness test. All testing sessions were performed with identical equipment, positioning, technique, and test order (Khorasani \& Bafghi, 2013).

A sprint is defined as any movement that reaches or exceeds the sprint threshold velocity for at least 1 second and any movement with an acceleration that occurs within the highest $5 \%$ of accelerations found in the corresponding velocity range (Dwyer \& Gabbet, 2012). Stationary 10-m sprint and flying 20-m sprint can be used to assess acceleration and maximal speed. Team sports requiring high intensity, an intermittent, non-continuous exercise that includes many sprints of different durations, rapid acceleration, jumping and agility. High-speed actions constitute the more crucial moments of the game and contribute directly to winning possession of the ball and scoring or conceding goals. The range of sprint distances recorded during games $(1.5105 \mathrm{~m})$ indicates the requirements of both acceleration and maximum speed, although players often initiate sprints when already moving at moderate speeds. (Alikhajeh, et al., 2012). Superior performance in varied speed tests of professional players compared to the general population and within higher soccer standard should indicate that certain speed attributes would be advantageous for elite soccer (Little \& Williams, 2003). 
The ability to exert force with the lower extremities quickly (Power= Force $x$ Velocity), is a predictor of disability among older adults, Lower leg power is an early indicator of poor function and higher leg power may indicate preserved physical function (Malik, et al., 2018). Horizontal and vertical jump tests used as the measure of power. The countermovement jump (CMJ) and 5 jump tests (5JT) were chosen as measures of functional leg power (Chaouachi, et al., 2010). Vertical jump performance was assessed using a portable force platform. Participants performed CMJ and squat jumps according to Bosco et al. Soccer is one of the most popular sports teams throughout the world, requiring high-intensity, an intermittent, non-continuous exercise that includes anaerobic performances such as power and etc. Power contributes to some of the total distance covered during a game that determines winning possession of the ball and scoring goals in soccer (Khorasani \& Bafghi, 2013). Selecting appropriate strength and power exercises that help increase the ability to develop force relative to BM and decrease ground contact time has been suggested to be a highly important aspect of training program design to improve printing speed (Barr, Sheppard, AgarNewman, \& Newton, 2014).

\section{Methodology}

\subsection{Participants}

Participants were 30 Under-18 school-level female netball players (age: $16.5 \pm 2.47$ years; height: $164.9 \pm 6.9 \mathrm{~cm}$; body mass: $60.1 \pm 6.6 \mathrm{~kg}$ ) who volunteered to participate in this study. All subjects were members of the Under-18 school netball squad of the school. Written consent was obtained from school administrators and parents before recruiting the subjects for the study. All subjects were starters in their team sports and participated in a regular training and competition schedule in team sports. During the competitive season, subjects trained 4-6 times a week. Subjects were accustomed to flexibility training (i.e., SS and DS) being part of their training routines. Testing procedures took place during the post-competitive phase of the season. None of the participants reported any current or ongoing neuromuscular diseases or musculoskeletal injuries specific to the ankle, knee, or hip joints. None of them were taking any dietary or performance supplements that might be expected to affect performance during the study. After receiving a detailed explanation of the potential benefits and risks associated with participation in the study, each participant signed an informed consent form before experimentation.

\subsection{Procedures}

The experimental research design was used to conduct the study. This study compared the effects of 3 different stretching warm-up protocols on sprint time, agility, and leg power relative to a control warm-up protocol without stretching. These warmups were designed based on their content (i.e., type of stretch, sequencing of static and dynamic stretching combinations, or exclusion of stretches). The three different stretching protocols are: (a) Static Stretching combined with Dynamic Stretching (SS+DS); (b) Dynamic Stretching combined with Static Stretching (DS+SS); (c) Dynamic Stretching combined with Dynamic Stretching (DS+DS). The agility, speed, and leg power tests provided functional measures to assess the effectiveness of these variables to provide suggestions for an optimal warm-up procedure. The experimental procedure is summarized in Figure 1 (Chaouachi, et al., 2010).

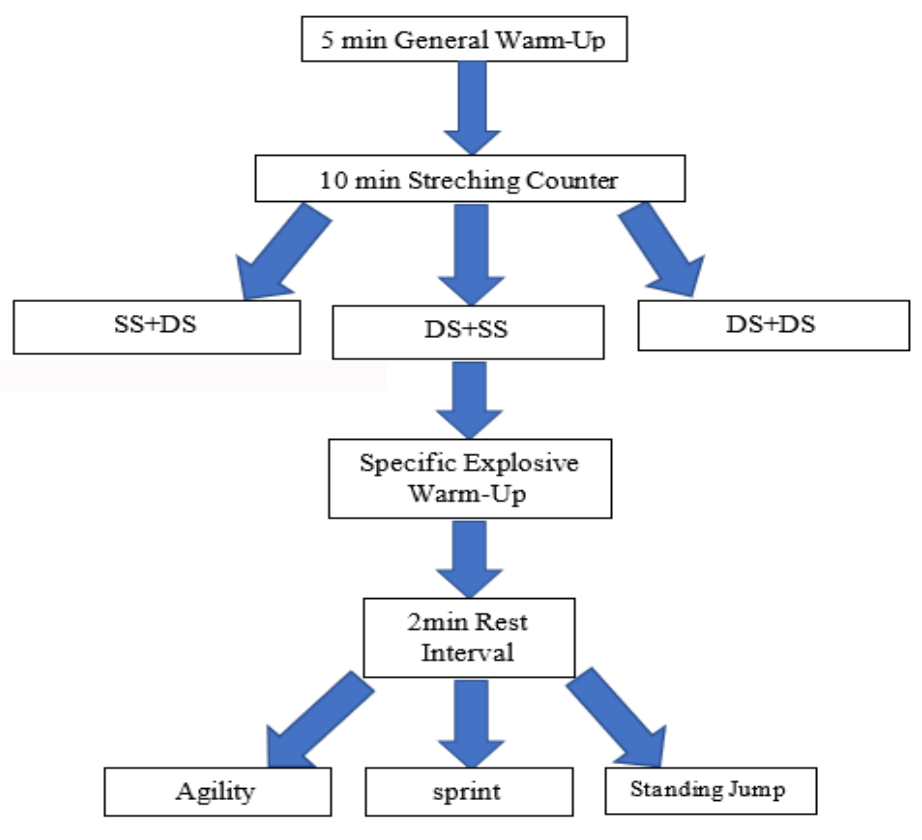

Fig. 1 : Experimental protocol 
Participants have attended a total of 4 data collection sessions except for an orientation session. Each subject was familiarized with the stretching exercises during the orientation phase and the three performance measures (30m sprint run, standing long jump, and T-drill). Each subject's age, height, and body mass were collected. The four sessions were completed during the subsequent eight days so that approximately 48 hours separated each test day. This procedure was chosen to minimize any performance changes that could occur over a longer period. The warm-up protocol assignment and the tests were counter balanced per person and per day to avoid carryover effects. All warm-ups with subsequent data collection and testing occurred in an indoor court to eliminate wind resistance or inclement weather. After completing one of the warm-up conditions, subjects proceeded to the performance testing sessions. The time between finishing the warm-up and beginning the performance testing was approximately 2 minutes. After the orientation session, all the participants attended the control session conducted without the stretching protocol and for the subsequent data collection and testing session. Three warm-up protocol assignments with different stretching protocols and the subsequent data collection and testing sessions were conducted after the control session. Before the warm-up protocol assignment with different stretching protocols, the participants were randomly divided into three groups. Every group was entitled to perform different warm-up protocols on three non-consecutive days. The warm-up protocol described by Chaouachi et al. (2010) was used in this study.

Table 1: Different warm up protocols during four non-continuous days.

\begin{tabular}{|c|c|c|c|c|c|c|c|c|c|}
\hline \multirow{3}{*}{$\begin{array}{l}\text { Protoc } \\
\text { ols }\end{array}$} & \multicolumn{9}{|c|}{ Days } \\
\hline & \multicolumn{3}{|c|}{ Group 1 } & \multicolumn{3}{|c|}{ Group2 } & \multicolumn{3}{|c|}{ Group 3} \\
\hline & $1^{s+}$ & $2^{\text {nad }}$ & $3^{\text {ead }}$ & $1^{\text {sh }}$ & $2^{\text {mit }}$ & $3^{\text {rd }}$ & $1^{\text {sa }}$ & $2^{\text {mal }}$ & $3^{\text {nt }}$ \\
\hline $\begin{array}{c}5 \text { min } \\
\text { Genera } \\
1 \\
\text { Warm- } \\
\text { up }\end{array}$ & + & + & + & + & + & + & + & + & + \\
\hline $10 \mathrm{~min}$ & SS & DS & DS & DS & DS & ss & DS & ss & DS \\
\hline Strechi & + & + & + & + & + & + & + & + & + \\
\hline $\mathrm{ng}$ & DS & ss & DS & ss & DS & DS & DS & DS & ss \\
\hline $\begin{array}{c}\text { Specifi } \\
c \\
\text { Explosi } \\
\text { ve } \\
\text { Warm } \\
U_{p}\end{array}$ & + & + & + & + & + & + & + & + & + \\
\hline $\begin{array}{l}2 \mathrm{~min} \\
\text { Rest }\end{array}$ & + & + & + & + & + & + & $\begin{array}{l}\text { Ac } \\
\text { Go }\end{array}$ & ofivate & $\begin{array}{c}\text { Yin } \\
\text { ngs td }\end{array}$ \\
\hline
\end{tabular}

(+) denotes activity included.

Source: Chaouachi, et al., 2010

The stretching warm-up consisted of 5 different stretching exercises designed to stretch the plantar flexors (principally gastrocnemius and soleus), hip flexors (quadriceps), hamstrings, hip extensors (gluteals), and adductors. Both SS and DS protocols stretched the same groups (Chaouachi, et al., 2010). The SS and DS exercises were repeated 2 times on each leg and were performed for 30 seconds with a 10-second recovery period between each exercise. No rest period was allowed when changing the limb. The stretching intensity was held at the maximal intensity condition. In the combined SS and DS protocols, the number of sets of SS and DS was reduced from 2 sets to 1 set for each muscle group of both legs to equalize the total time of stretching.

\subsection{Data Gathering Techniques}

Three main tests: the T-test, 30m sprint test and standing long jump, were used to evaluate the performance of three bio-motor abilities agility, speed, and leg power of netball players, respectively. Maximum value from the 3 test trials of all measurements was used for analysis. 
Statistical analysis was conducted by using SPSS. The level of significance was set at $5 \%$ for the analysis. Two-way (order $x$ protocol) ANOVA was used to determine the effects of stretching protocols on agility, sprinting, and leg power of the participants.

\section{Results and Discussion}

No significant interactions were found between order and protocol for all agility, speed and leg power performance of the participants $(p>0.05)$.

According to current findings illustrated in Table 2 and Figures 2, 3, and 4, there is no significant differences among the stretching protocols $(S S+D S, D S+S S, D D+D D)$ regarding agility $(p=0.257)$, speed $(p=0.106)$, and leg power $(p=0.902)$ performance of the participants.

Table 2. Significance and descriptive statistics of protocol type effect

\begin{tabular}{|c|c|c|c|c|c|c|}
\hline \multirow{2}{*}{$\begin{array}{l}\text { Dependent } \\
\text { Variables }\end{array}$} & \multirow[b]{2}{*}{ Test } & \multirow[b]{2}{*}{$\begin{array}{c}\text { Sig } \\
\text { Value }\end{array}$} & \multicolumn{4}{|c|}{ Protocol Type } \\
\hline & & & No-Stretch & SS+DS & $\mathrm{DS}+\mathrm{SS}$ & $\mathrm{DS}+\mathrm{DS}$ \\
\hline Agility & T-Test & 0.257 & $9.11 \pm 0.44$ & $8.70 \pm 1.67$ & $9.10 \pm 0.44$ & $8.93 \pm 0.44$ \\
\hline Speed & $\begin{array}{l}30 \mathrm{~m} \\
\text { Sprint }\end{array}$ & 0.106 & $4.25 \pm 0.28$ & $4.40 \pm 0.31$ & $4.41 \pm 0.37$ & $4.25 \pm 0.35$ \\
\hline $\begin{array}{c}\text { Leg } \\
\text { Power }\end{array}$ & $\begin{array}{c}\text { Standing } \\
\text { Long } \\
\text { Jump }\end{array}$ & 0.902 & $126.23 \pm 12.71$ & $127.44+12.61$ & $124.91 \pm 12.87$ & $118.02 \pm 33.24$ \\
\hline
\end{tabular}

* $\mathrm{p} \leq 0.05$

Source: Author, 2020

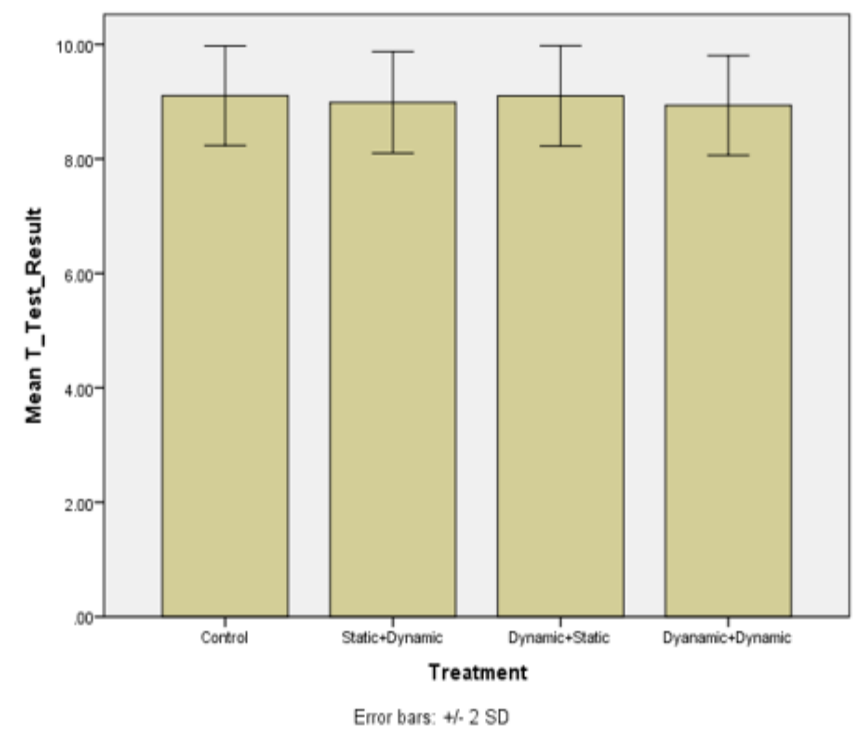

Figure 2: Results of T-test

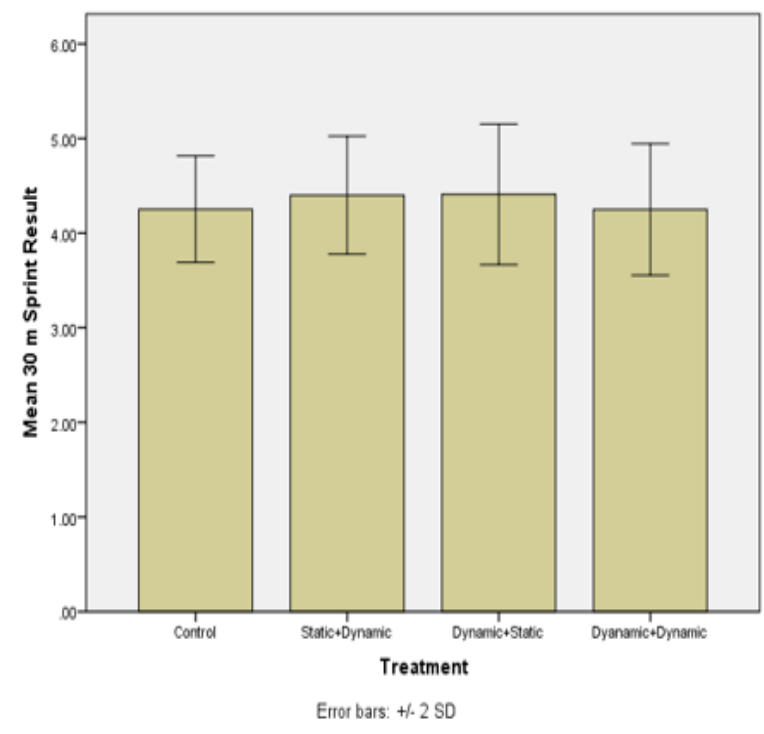

Figure 3: Results of 30m Sprint 


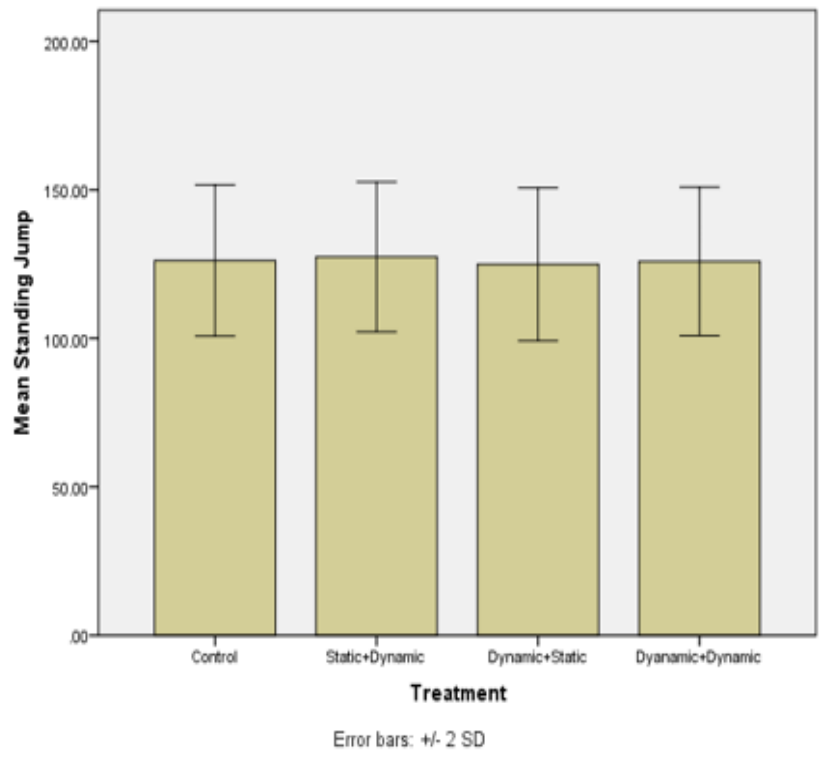

Figure. 4: Results of standing long jump

The purpose of the current study was to identify the effect of sequencing lower-body static and dynamic stretching combinations (SS+DS, DS+SS, DS+DS) on the agility, speed, and leg power performance of school-level female netball players. The most exciting findings of this research were that in training, the sequence of static and dynamic stretching combination (SS+DS, DS+SS, DS+DS) did not significantly affect agility, speed, and leg power of school-level netball athletes. Although the majority of studies report static stretching-induced impairments, some studies have shown no deficits in running economy, sprint time, and jump performance. Nevertheless, DS or ballistic stretching has been reported to enhance performance in leg power, agility, speed, and vertical jump. Alternatively, other studies have reported no change in MVC force, countermovement, and drop jump heights with prior dynamic stretching (Chaouachi, et al., 2010).

Warming-up can result in decreased muscle viscosity, increased variables such as oxygen uptake during subsequent exercise, nerve conduction velocity, glycolysis, ROM, anaerobic performance, and tensile muscle strength. Hence, the faster sprint times in the control condition may be attributed to the increased muscle temperature without the interference of stretching. (Chaouachi, et al., 2010).

\section{Conclusion}

The results of the analysis allow the author to retain the hypothesis that a sequence of static and dynamic stretching combinations (SS+DS, DS+SS, DS+DS) does not significantly affect the agility, speed, and leg power performance of school-level female netball players. Warm-up is usually thought to positively affect injury prevention for any sports activity. There is a need to conduct more researches on the effect of the stretching protocol on agility, sprint, and leg power with diverse athlete populations representing different sports, increased sample sizes and longer durations of protocols. Further, better-controlled researches are required to interpret the mechanisms responsible for changes in performance following any types of stretching protocol for the various sports performance components. More research studies should be conducted related to the warm-up protocols by taking non-trained participants and various environments.

Funding: This research was not funded by any funding agency.

Acknowledgments: Researchers acknowledge all participants of the study and school administrators.

Conflicts of Interest: The authors declare no conflicts of interest. 


\section{References}

[1] Ahsan, M., \& Mohammad, A. (2018). Effects of Different Warm-Up Techniques on Dynamic Balance and Muscular Strength. European Journal of Physical Education and Sport Science, 4(12).

[2] Pojskic, H., Pagaduan, J., Uzicanin, E., \& Babajic, F. (2015). Acute Effects of Loaded Whole Body Vibration Training on Performance. Asian J Sports Med.

[3] Ab Malik, Z. I. (2018). Effects of Different Intensities of Weighted Vest Warming up Session on Physical Performances of Power Sports Athletes. International Journal of Engineering \& Technology, 161-164.

[4] Alikhajeh , Y., Rahimi, N. M., \& Fazeli, H. (2012). Differential Stretching Protocols During Warm Up on Select Performance Measures for Elite Male Soccer Players. Procedia - Social and Behavioral Sciences, 46, 1639 - 1643.

[5] Chaouachi, A., Castagna, C., Chtara, M., \& Brughelli, M. (2010). Effect Of Warm-Ups Involving Static Or ynamic Streching on Agility,Sprinting and Jumping Performance In Trained Individuals. Journal of Strength and Conditioning Research the TM, 24, 2001-2011.

[6] Dwyer, D. B., \& Gabbet, T. J. (2012). Global Positioning System Data Analysis:Velocity Ranges and a New Definition Of Sprinting For Feild Sport Athletes. Strength and Conditioning Research, 26(3), 818-824.

[7] Hopper, D., Lo, S. K., Kirkham, C., \& Elliott, B. (1992). Landing Patterns in Netball: Analysis of an International Game. Br J Sp Med, 26(2).

[8] Khorasani, M. A., \& Bafghi, A. F. (2013). Sustaining Effect of Different Stretching Methods on Power and Agility after Warm-Up Exercise in Soccer Players. World Applied Sciences Journal, 520-525.

[9] Leon, C. O.-J. (2012). A purposeful dynamic stretching routine. Strategies, 16-19.

[10] Macmillian, D. J., Moore, J. H., Hatler, B. S., \& Taylor, D. C. (2006). Dynamic vs Static-Streching Warm up: The Effect on Power and Agility Performan. Strength and Conditioning, 492-499.

[11] Malik, Z. A., Asmadi I, s., Rosman, N. A., Osman, N., \& Mahmod, A. K. (2018). Effects of Different Intensities of Weighted Vest Warming up Session on Physical Performances of Power Sports Athletes. International Journal of Engineering \& Technology, 161-164.

[12] McMillian, D. M. (2006). Dynamic vs. static stretching warm-up: The effect on power and agility performance. Journal of Strength and Conditioning Research, 492-499.

[13] Ogura, Y. M. (2007). Duration of static stretching influences muscle force production in hamstring muscles. Journal of Strength and Conditioning Research, 788-792.

[14] Plisk, S. (2000). Speed, agility, and speed-endurance development. Essentials of strength training and conditioning, 471-491.

[15] Shrier, M. (2004). Does stretching improve performance? A systematic and critical review of the literature. Clinical Journal of Sports Medicine. [16] Barr, M. J., Sheppard, J. M., Agar-Newman, D. J., \& Newton, R. U. (2014). Transfer effect of strength and power training to the sprinting kinematics of international rugby players. The Journal of Strength \& Conditioning Research, 28(9), 2585-2596.

[17] Little, T., \& Williams, A. (2003). Specificity of acceleration, maximum speed and agility in professional soccer players (pp. pp-144). London, UK: Routledge. 